\title{
1 Quantitative Spectroscopic Analysis of Heterogeneous Mixtures: the Correction of Multiplicative Effects 3 Caused by Variations in Physical Properties of Samples
}

5 Jing-Wen Jin $^{a}$, Zeng-Ping Chen ${ }^{* a}$, Li-Mei Li ${ }^{a}$, Raimundas Steponavicius ${ }^{b}$, Suresh N. Thennadil ${ }^{c}$, Jing Yang $^{a}$ and Ru-Qin $Y u^{* a}$

a. State Key Laboratory of Chemo/Biosensing and Chemometrics, College of Chemistry and Chemical

$14 *$ Corresponding author

15 Tel.: (+86) 731 88821916; Fax: $(+86) 73188821916$;

16 E-mail Address: zpchen2002@hotmail.com (Z.P. Chen), rqyu@hnu.cn (R.Q. Yu) 
18 ABSTRACT: Spectral measurements of complex heterogeneous types of mixture samples are often

19 affected by significant multiplicative effects resulting from light scattering, due to physical variations

20 (e.g. particle size and shape, sample packing and sample surface, etc.) inherent within the individual 21 samples. Therefore, the separation of the spectral contributions due to variations in chemical 22 compositions from those caused by physical variations is crucial to accurate quantitative spectroscopic 23 analysis of heterogeneous samples. In this work, an improved strategy has been proposed to estimate the 24 multiplicative parameters accounting for multiplicative effects in each measured spectrum, and hence mitigate the detrimental influence of multiplicative effects on the quantitative spectroscopic analysis of heterogeneous samples. The basic assumption of the proposed method is that light scattering due to physical variations has the same effects on the spectral contributions of each of the spectroscopically active chemical component in the same sample mixture. Based on this underlying assumption, the proposed method realizes the efficient estimation of the multiplicative parameters by solving a simple quadratic programming problem. The performance of the proposed method has been tested on two

31 publicly available benchmark data sets (i.e. near-infrared total diffuse transmittance spectra of four-component suspension samples and near infrared spectral data of meat samples) and compared with some empirical approaches designed for the same purpose. It was found that the proposed method provided appreciable improvement in quantitative spectroscopic analysis of heterogeneous mixture samples. The study indicates that accurate quantitative spectroscopic analysis of heterogeneous mixture samples can be achieved through the combination of spectroscopic techniques with smart modeling methodology. 


\section{1. Introduction}

44 The quantitative analysis of heterogeneous mixture samples using conventional instruments such as

45 HPLC generally involves troublesome and time-consuming sample preparations. Due to their high

46 measuring speed, multiplicity of analysis, non-destructivity, flexibility and especially requirement of

47 less or even no sample preparations, spectroscopic technologies such as near infrared (NIR), mid

48 infrared (MIR) and Fourier-transform Raman spectroscopy (FT-Raman) have been increasingly applied

49 to the analysis of complex systems in areas of chemicals, food processing, agriculture and

50 pharmaceuticals, etc ${ }^{1-6}$. However, when analyzing complex heterogeneous mixture samples that exhibit

51 sample-to-sample variability in physical properties using spectroscopic instrumentation, the

52 multiplicative light scattering effects caused by the uncontrolled variations in optical path length due to

53 the physical differences between samples (e.g. particle size and shape, sample packing, and sample

54 surface, etc) would 'scale' the entire spectral measurement and hence mask the spectral variations

55 relating to the content differences of chemical compounds in the samples ${ }^{7}$. The presence of dominant

56 multiplicative effects in spectral data could invalidate the underlying assumption of commonly used

57 multivariate linear calibration methods such as $\mathrm{PCR}^{8}$ and $\mathrm{PLS}^{9}$ which postulates a linear relationship

58 between spectral measurements and the contents of chemical components, and hence significantly

59 deteriorate the predictive performance of calibration models built by multivariate linear calibration

60 methods. The separation of the spectral contributions due to variations in chemical compositions from

61 those caused by multiplicative effects is therefore crucial to the accurate quantitative analysis of messy

62 spectral data with multiplicative effects. 
63 A number of chemometric pre-processing methods, e.g., Multiplicative Signal Correction (MSC) ${ }^{7}$,

64 Standard Normal Variate (SNV) ${ }^{10}$, Inverted Signal Correction (ISC) ${ }^{11}$, Extended Inverted Signal

65 Correction (EISC) ${ }^{12}$, Extended MSC (EMSC) ${ }^{13}$ and Modified EMSC ${ }^{14}$ have been proposed to remove

66 the multiplicative effects caused by variations in physical properties of samples. However MSC, ISC

67 and EISC could only be applied to a spectrum that has wavelength regions containing no chemical

68 information, i.e. influenced only by the multiplicative effects. Otherwise, they could result in

69 dramatically poor results. The applicability of EMSC and the modified EMSC is limited due to the

70 requirement of the pure spectra for all spectroscopically active chemical components present in the

71 samples which is difficult to satisfy in practice.

72 Recently, Thennadil et al. proposed an interesting approach for the correction of multiple light

73 scattering effects by making use of radiative transfer theory ${ }^{15-16}$. Though this approach can to some

74 extent improve the predictive performance of multivariate calibration models, its implementation

75 complexity and the requirement of three measurements for each mixture sample (i.e. total diffuse

76 transmittance, total diffuse reflectance and collimated transmittance) make it difficult to use in practice.

77 More recently in a review of pharmaceutical applications of separation of absorption and scattering in

78 near-infrared spectroscopy, similar concepts to the approach mentioned above are discussed ${ }^{17}$. Another

79 similar approach to compensate for the scattering effects in reflectance spectroscopy was developed by

80 Kessler et al. by integrating Kubelka-Munk equation with multivariate curve resolution (MCR) ${ }^{18}$. Like

81 the method based on radiative transfer theory, the application of hard model constrained MCR-ALS

82 algorithm is dependent on the availability of two measurements for each mixture sample (i.e. the diffuse 
83 reflectance spectra of a sample with an optically infinite thickness and a sample of finite thickness).

84 Hence the scope of its applicability is also limited.

85 To overcome these limitations, one of the present authors developed a novel multiplicative effect

86 correction approach, Optical Path-Length Estimation and Correction (OPLEC) ${ }^{19,20}$. OPLEC adopted

87 the following two-step procedure for the correction of multiplicative effects in spectral measurements.

88 First of all, the multiplicative parameters accounting for multiplicative effects in the spectral

89 measurements of the calibration samples are estimated by a unique method deduced solely from the

90 linear transformation of the calibration spectral measurements. And then the multiplicative effects in the

91 spectral measurements of the test samples are efficiently removed by a dual-calibration strategy.

92 Without placing any requirement on the spectral measurements, OPLEC can efficiently separate the

93 multiplicative effects of samples' physical properties from the spectral variations related to the chemical

94 compositions, and hence has much wider applicability than other methods reported in the literature. The

95 development of OPLEC provided an important contribution to the solution of multiplicative light

96 scattering issues. Whereas the first step of OPLEC, i.e. the estimation of the multiplicative parameters

97 for the calibration samples, involves the determination of the number of spectroscopically active

98 chemical components in the systems under study. A poor estimation of the number of chemical

99 components would result in suboptimal performance of OPLEC. For complex systems, the estimation of

100 the number of chemical components is not a trivial task. Therefore, the OPLEC method needs to be

101 refined to realize its full potential for spectroscopic quantitative analysis of heterogeneous mixtures.

102 The objectives of this study were (1) to redesign the method in OPLEC for the estimation of the

103 multiplicative parameters for the spectral measurements of the calibration samples, (2) to develop a 
104 simple but effective approach for determining the optimal model parameter (i.e. the number of 105 spectroscopically active chemical components) in OPLEC, (3) to improve the robustness of OPLEC 106 when being applied to complex systems, and finally (4) to evaluate the performance of the modified 107 OPLEC method on two publicly available benchmark data sets.

\section{Theory}

1112.1 The dual calibration strategy adopted by OPLEC to correct multiplicative effects

112 For spectral measurements with multiplicative effects caused by changes in the optical path-length due

113 to the physical variations of the samples, the measured spectrum ( $\mathbf{x}_{i}$, row vector) of sample $i$ composed

114 of $J$ chemical components can be approximated by the following model ${ }^{6,7,21}$ :

$$
\mathbf{x}_{i}=p_{i} \sum_{j=1}^{J} c_{i, j} \mathbf{s}_{j}, \quad i=1,2, \ldots, I
$$

115 Where $c_{i, j}$ is the concentration of the $j$-th chemical component in the $i$-th mixture sample; $\mathbf{s}_{j}$ represents 116 the pure spectrum of $j$-th chemical component in the mixtures. The coefficient $p_{i}$ accounts for the 117 multiplicative effects in the spectral measurements of the $i$-th sample caused by changes in the optical 118 path-length due to the physical variations of the sample; $I$ denotes the number of calibration samples.

119 Assume the first component is the target constituent in the mixtures and $\sum_{j=1}^{J} c_{i, j} \mathbf{s}_{j}=1$ (which strictly 120 hold for $c_{i, j}$ representing unit-free concentration such as weight fraction and mole fraction), then eq.1 121 can also be expressed as: 


$$
\mathbf{x}_{i}=p_{i} c_{i, 1} \Delta \mathbf{s}_{1}+p_{i} \mathbf{s}_{2}+\sum_{j=3}^{J} p_{i} c_{i, j} \Delta \mathbf{s}_{j}, \Delta \mathbf{s}_{j} \Rightarrow \mathbf{s}_{j}-\mathbf{s}_{2}
$$

122 It is obvious that a linear relationship exists between $\mathbf{x}_{i}$ and $p_{i}$, and also between $\mathbf{x}_{i}$ and $p_{i} c_{i, 1}$. It should

123 be noted that this conclusion would also hold when the content of one constituent (or matrix substances)

124 does not vary over mixture samples. Provided the multiplicative parameter vector $\mathbf{p}\left(\mathbf{p}=\left[p_{1} ; p_{2} ; \ldots ; p_{I}\right]\right)$

125 for the calibration samples is available (actually it can be estimated from the calibration spectra by the

126 multiplicative parameter estimation method outlined in section 2.2), two following calibration models

127 can therefore be built by multivariate linear calibration methods such as PLS. The first model is between

$128 \mathbf{X}\left(\mathbf{X}=\left[\mathbf{x}_{1} ; \mathbf{x}_{2} ; \ldots ; \mathbf{x}_{I}\right]\right)$ and $\mathbf{p}$, and the other is between $\mathbf{X}$ and $\operatorname{diag}\left(\mathbf{c}_{1}\right) \mathbf{p}$

$129\left(\operatorname{diag}\left(\mathbf{c}_{1}\right) \mathbf{p}=\left[p_{1} \times c_{1,1} ; p_{2} \times c_{2,1} ; \ldots ; p_{I} \times c_{I, 1}\right]\right)$. For simplicity, the same number of latent components is

130 generally used in the above two PLS calibration models. Once the spectrum of a test sample has been

131 recorded, the content of the target constituent in the test sample can then be obtained by dividing the

132 prediction of the second calibration model by the corresponding prediction of the first calibration model.

\subsection{Multiplicative parameter estimation}

135 Obviously, the estimation of the multiplicative parameter vector $\mathbf{p}$ for the calibration samples is the key

136 to the correction of the multiplicative effects by the above dual calibration strategy. The performance of

137 the multiplicative parameter estimation method in the original OPLEC method ${ }^{19}$ relies on the accurate

138 estimation of the number of spectroscopically active chemical components in the systems under study.

139 Poor estimation of the number of chemical components could significantly affect the performance of 
140 OPLEC. With a view to improve the robustness of OPLEC, the following refined method for the

141 estimation of multiplicative parameter vector $\mathbf{p}$ for the calibration samples was proposed in this work.

142 Suppose the singular value decomposition of $\mathbf{X}\left(\mathbf{X}=\left[\mathbf{x}_{1} ; \mathbf{x}_{2} ; \ldots ; \mathbf{x}_{I}\right]\right)$ can be expressed as follows:

$$
\mathbf{X}=\left[\mathbf{U}_{s}, \mathbf{U}_{n}\right]\left[\begin{array}{cc}
\sum_{s} & 0 \\
0 & \sum_{n}
\end{array}\right]\left[\mathbf{V}_{s}, \mathbf{V}_{n}\right]^{\mathrm{T}}=\mathbf{U}_{s} \sum_{s} \mathbf{V}_{s}^{\mathrm{T}}+\mathbf{E}
$$

143 Where, $\mathbf{E}=\mathbf{U}_{n} \sum_{n} \mathbf{V}_{n}^{\mathrm{T}}$; superscript ' $\mathrm{T}$ ' denotes the transpose; subscripts 's' and 'n' signify that the 144 corresponding factors represent spectral information and noise, respectively. Suppose the actual number 145 of spectroscopically active chemical components in the system studied is $r$, then both $\mathbf{U}_{\mathrm{s}}$ and $\mathbf{V}_{\mathrm{s}}$ consist 146 of $r$ columns. According to eq.2, both vectors $\mathbf{p}$ and $\operatorname{diag}\left(\mathbf{c}_{1}\right) \mathbf{p}$ are in the column space of $\mathbf{U}_{\mathrm{s}}$, so the 147 following equations hold:

$$
\begin{aligned}
& \mathbf{U}_{s} \mathbf{U}_{s}^{\mathrm{T}} \mathbf{p}=\mathbf{p} \\
& \mathbf{U}_{s} \mathbf{U}_{s}^{\mathrm{T}} \operatorname{diag}\left(\mathbf{c}_{1}\right) \mathbf{p}=\operatorname{diag}\left(\mathbf{c}_{1}\right) \mathbf{p}
\end{aligned}
$$

148 Since there is no requirement to know the absolute value of $p_{i}, p_{i}$ can be assumed to be no less than 149 unity $(\mathbf{p} \geq \mathbf{1})$. Therefore, the vector $\mathbf{p}$ satisfying equations 4 and 5 can be obtained by solving the 150 following constrained optimization problem:

$$
\min _{\mathbf{p}} \frac{1}{2}\left(\left\|\mathbf{U}_{s} \mathbf{U}_{s}^{\mathrm{T}} \mathbf{p}-\mathbf{p}\right\|_{2}^{2}+\frac{1}{w^{2}}\left\|\mathbf{U}_{s} \mathbf{U}_{s}^{\mathrm{T}} \operatorname{diag}\left(\mathbf{c}_{1}\right) \mathbf{p}-\operatorname{diag}\left(\mathbf{c}_{1}\right) \mathbf{p}\right\|_{2}^{2}\right), \quad \text { subject to } \mathbf{p} \geq 1
$$

151 Where, \|\|$_{2}$ denotes $l^{2}$ norm; $w$ is a weight to balance the two parts in the above optimization function. 152 It can be simply set to be the maximum element of $\mathbf{c}_{1}$. The above constrained optimization problem can 
153 be transformed into an equivalent quadratic programming problem (which can be resolved by the 154 quadprog function in MATLAB. The MATLAB code for the multiplicative parameter estimation 155 method is available in Supporting Information):

$$
\min _{\mathbf{p}} f(\mathbf{p})=\frac{1}{2} \mathbf{p}^{\mathrm{T}}\left(\left(\mathbf{I}-\mathbf{U}_{s} \mathbf{U}_{s}^{\mathrm{T}}\right)+\operatorname{diag}\left(\mathbf{c}_{1} / w\right)\left(\mathbf{I}-\mathbf{U}_{s} \mathbf{U}_{s}^{\mathrm{T}}\right) \operatorname{diag}\left(\mathbf{c}_{1} / w\right)\right) \mathbf{p}, \text { such that }-\mathbf{p} \leq-1
$$

\subsection{Determination of the number of columns in $\boldsymbol{U}_{s}$}

158 Theoretically, the number of columns in $\mathbf{U}_{\mathrm{s}}$ (i.e. parameter $r$ ) should equal to the number of 159 spectroscopically active chemical components in the systems under study. It is generally difficult to 160 determine the exact number of spectroscopically active chemical components in a complex system.

161 Moreover, when the spectral data does not strictly obey the model in eq. 1, the optimal number of 162 columns in $\mathbf{U}_{\mathrm{s}}$ might not solely depend on the number of spectroscopically active chemical components 163 in the system under study, which would further complicate the situation. Fortunately, a simple 164 mathematical analysis reveals that $\min _{\mathbf{p}} f(\mathbf{p})$ decreases dramatically with the increase of $r$ at the very 165 start, and then tends to be steady when $r$ exceeds certain threshold value. Therefore, the optimal value of $166 r$ can be determined by locating the turning point in the plot of $\min _{\mathbf{p}} f(\mathbf{p})$ versus $r$.

\section{Case studies}

170 The effectiveness of the modified OPLEC method (hereafter referred to OPLEC $m$ ) with respect to its

171 ability to estimate multiplicative parameters was first tested on the near-infrared total diffuse 172 transmittance spectra of four-component suspension system consisting of water, deuterium, ethanol, and 
173 polystyrene (hereafter referred to four-component suspension data). To further explore the potential of 174 OPLEC $_{\mathrm{m}}$, another real-world near-infrared transmittance spectra of meat samples recorded on a Tecator 175 Infratec Food and Feed Analyzer (hereafter referred to tecator data) is employed. This spectral data set 176 is publicly available and hence ensures that the interested reader can repeat the analysis.

\subsection{Four-component suspension data ${ }^{16}$}

179 The four-component suspension system is composed of three fully miscible absorbing species of water, 180 deuterium oxide and ethanol and a species that both absorbs and scatters light (i.e., a particulate species 181 of polystyrene). Specifically, the range of particle size and concentration were chosen to be $100 \sim 500 \mathrm{~nm}$ 182 and $1 \sim 5 \mathrm{wt} \%$, respectively, such that the following conditions were satisfied: stable suspension, multiple 183 scattering, and sufficient signals in measurement. A total of 42 samples were prepared using various 184 combinations of the concentrations of the four components and particle sizes of which the total diffuse 185 transmittance $\left(\mathbf{T}_{\mathrm{d}}\right)$ spectra were recorded on a scanning spectrophotometer (CARY 5000) fitted with a 186 diffuse reflectance accessory (DRA-2500). The spectral data were collected in the wavelength region of 187 1500-1880 nm with an interval of $2 \mathrm{~nm}$, resulting in measurements at 191 discrete wavelengths per 188 spectrum. Twenty-two suspension samples' spectra were randomly selected to construct the calibration 189 data set. The remaining twenty spectra from the other suspension samples made up the test data set. The 190 absorbing-only species of deuterium oxide with concentration range between $20 \%$ and $58 \mathrm{wt} \%$ was 191 taken as the analyte of interest in the present analysis and all the total diffuse transmittance spectra were 192 transformed into absorbance spectra prior to the analysis. More experimental details can be found in the 193 original paper of Steponavicius and Thennadi ${ }^{16}$. 
196 This benchmark spectral data set consists of the near-infrared absorbance spectra of 240 meat samples 197 recorded on a Tecator Infratec Food and Feed Analyzer working in the wavelength range 850-1050 nm 198 with an interval of $2 \mathrm{~nm}$ by the Near Infrared Transmission principle. Each sample contains finely 199 chopped pure meat with different moisture, fat and protein contents. A Soxhlet method was used as the 200 laboratory reference for fat determination. The Soxhlet values ranged from $2 \%$ to $59 \%$ fat. The 240 201 spectra were divided into 5 data sets for the purpose of model validation and extrapolation studies 202 (calibration set: 129; validation set: 43; test set: 43; extrapolation set for fat: 8; extrapolation set for 203 protein: 7). The task in the present work is restricted to predict the fat content (\%) of a meat sample on 204 the basis of its near infrared absorbance spectrum, the extrapolation set for protein is therefore excluded. 205 The tecator data is available at http://lib.stat.cmu.edu/datasets/tecator.

\subsection{Data pre-treatment}

208 For the aforementioned two data sets, the possible additive baseline effects and wavelength dependent 209 spectral variations were firstly removed by projecting the measured spectra onto the orthogonal 210 complement of the space spanned by the row vectors of $\mathbf{M}=\left[\mathbf{1} ; \boldsymbol{\lambda} ; \boldsymbol{\lambda}^{2}\right]^{19}$. The pre-processed spectra 211 were then used to calculate the multiplicative parameter vector $\mathbf{p}$ for the calibration samples. The dual 212 calibration models in OPLEC $_{m}$ were built on the pre-processed spectra by using PLS method. The 213 predictive performance of OPLEC $_{m}$ was compared with those of PLS calibration models with and 214 without the application of data preprocessing methods such as MSC, SNV, EISC and EMSC as long as 
215 they are applicable. The root-mean-square error of prediction (RMSEP) was used to assess the 216 performance of the calibration models.

\section{Results and discussion}

\subsection{Four-component suspension data}

221 The raw total transmittance spectra of the four-component suspension samples are presented in Figure 1.

222 It can be observed that the variations in polystyrene particle size and concentration across samples

223 resulted in significant additive baseline shift as well as multiplicative effects in the spectral data.

224 Though the additive baseline effects and possible wavelength dependent spectral variations can be 225 readily removed by orthogonal projection pre-processing, the multiplicative effects as a consequence of 226 the changes in sample's effective optical path-length are rather difficult to correct. Such multiplicative 227 effects can not be effectively modeled by multivariate linear calibration models either. Without being 228 properly corrected or modeled, they can significantly deteriorate the predictive performance of 229 multivariate linear calibration models ${ }^{13,19}$. 


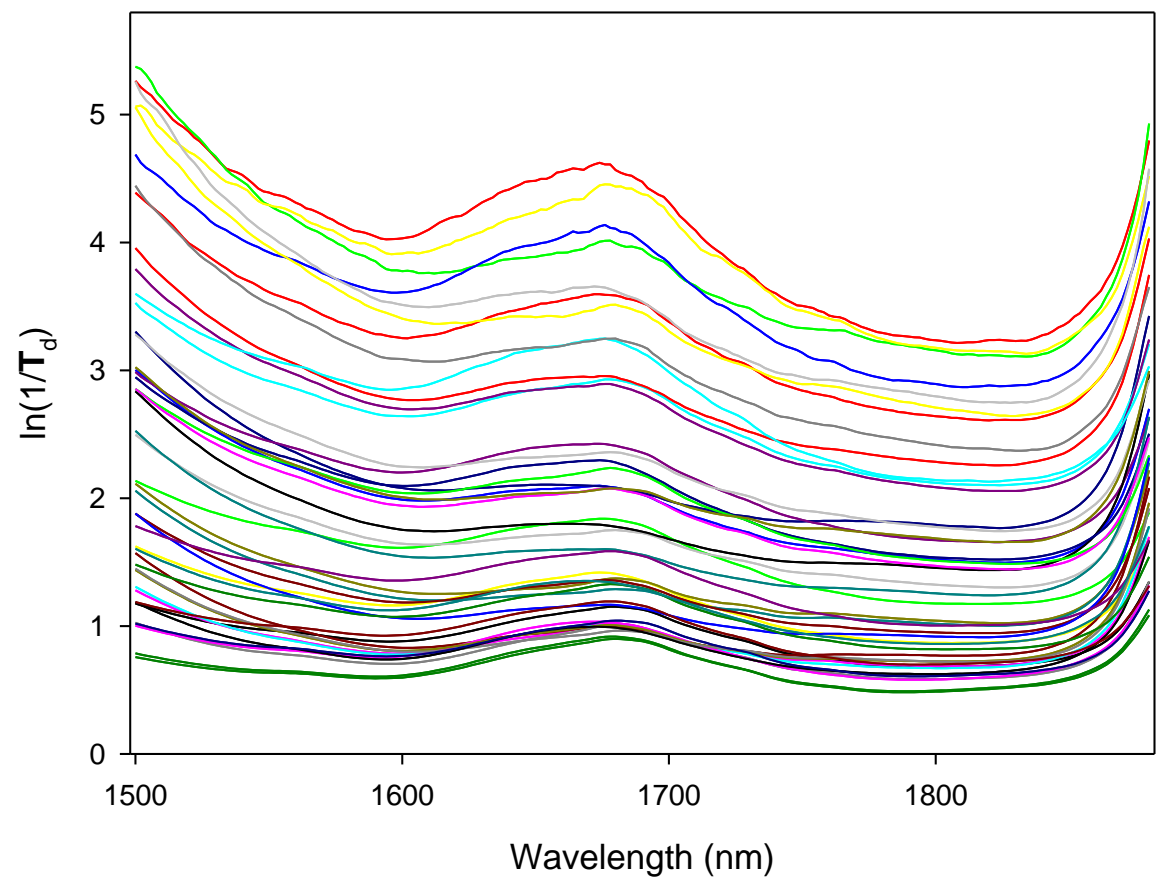

230

Wavelength $(\mathrm{nm})$

231 Figure 1: The raw spectra of the four component suspension system. 
232 As stated in the theory section, $\mathrm{OPLEC}_{\mathrm{m}}$ can effectively correct the multiplicative effects in spectral 233 measurements. OPLEC $\mathrm{m}_{\mathrm{m}}$ consists of two main steps. The first step is to estimate the multiplicative 234 parameter vector $\mathbf{p}$ for the calibration samples from the orthogonal projection pre-processed spectra. 235 The estimation of the multiplicative parameter vector $\mathbf{p}$ for the calibration samples requires the 236 determination of the actual number of spectral variation sources $(r)$ in the calibration spectra, which can 237 be achieved by scrutinizing the plot of $\min _{\mathbf{p}} f(\mathbf{p})$ versus $r$ (Figure 2). From Figure 2, it can be seen that $238 \min _{\mathbf{p}} f(\mathbf{p})$ decreases obviously when the number of columns of $\mathbf{U}_{\mathrm{s}}$ increases from one to three and 239 including more components in $\mathbf{U}_{s}$ leads to no significant changes in $\min _{\mathbf{p}} f(\mathbf{p})$, which means the most 240 spectral information relevant to $\mathbf{p}$ and $\operatorname{diag}\left(\mathbf{c}_{1}\right) \mathbf{p}$ was included in the first three principal components of $241 \mathbf{U}_{\mathrm{s}}$. Therefore, the optimal value of $r$ was then set to three. 


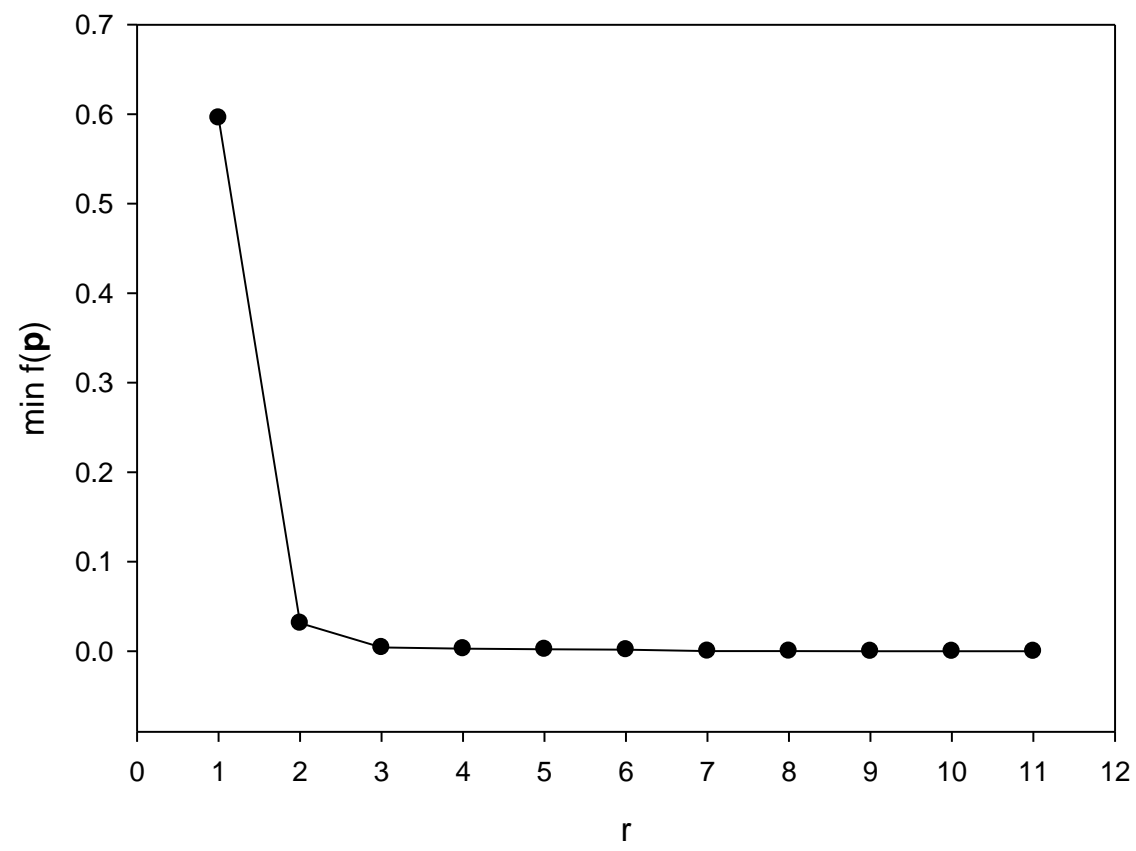

244

245 Figure 2: The relationship between $\min _{\mathbf{p}} f(\mathbf{p})$ and the number of columns of $\mathbf{U}_{\mathrm{s}}($ i.e. $r$ ) for the four 246 component suspension data. 
After the estimation of the multiplicative parameter vector $\mathbf{p}$ for the calibration samples, one can assess the applicability of $\mathrm{OPLEC}_{\mathrm{m}}$ to the spectral data set by examining the two plots of $\mathbf{p}$ vs $\mathbf{U}_{s} \mathbf{U}_{s}^{T} \mathbf{p}$ and $\operatorname{diag}\left(\mathbf{c}_{1}\right) \mathbf{p}$ vs $\mathbf{U}_{s} \mathbf{U}_{s}^{T} \operatorname{diag}\left(\mathbf{c}_{1}\right) \mathbf{p}$, respectively (supporting information, Figure S-1). As shown in Figure S-1, both $\mathbf{p}$ and $\operatorname{diag}\left(\mathbf{c}_{1}\right) \mathbf{p}$ are in good agreement with $\mathbf{U}_{s} \mathbf{U}_{s}^{T} \mathbf{p}$ and $\mathbf{U}_{s} \mathbf{U}_{s}^{T} \operatorname{diag}\left(\mathbf{c}_{1}\right) \mathbf{p}$, 251 respectively, which confirms that a linear relationship exists between $\mathbf{x}_{i}$ and $p_{i}$, and also between $\mathbf{x}_{i}$ and $252 p_{i} c_{i, 1}$. The dual calibration strategy of OPLEC $_{\mathrm{m}}$ is therefore applicable to the four component 253 suspension data. Figure S-1 also reveals the presence of significant variations of multiplicative effects 254 ( $p_{i}$ varying from 1 to 3.09) in the calibration samples. Multiplicative effect correction methods such as $255 \mathrm{OPLEC}_{\mathrm{m}}$ are therefore needed to remove such significant multiplicative effects in the spectral 256 measurements.

257 Figure $3 \mathrm{a}$ compared the predictive performance of the optimal OPLEC $_{\mathrm{m}}$ calibration model for 258 deuterium oxide and the corresponding optimal PLS models with and without the application of 259 preprocessing methods (e.g. SNV, MSC, EISC and EMSC). Obviously, as a result of the presence of 260 severe multiplicative effects, PLS calibration model built on the raw calibration spectra could not give 261 satisfactory predictions for the deuterium oxide in the test suspension samples. Preprocessing the 262 calibration spectra by MSC, SNV or EISC can, to some extent, improve the predictive performance of 263 PLS calibration models in terms of RMSEP values. However, due to the lack of a wavelength region 264 containing no chemical information in the spectral data, the multiplicative effects can not be fully 265 corrected by MSC, SNV or EISC. Hence, the predictive errors of the PLS calibration models built on 266 the calibration spectra pre-processed by MSC, SNV and EISC are still comparatively high. As expected, 267 OPLEC $_{\mathrm{m}}$ offers the best improvement in terms of the predictive ability among all the pre-processed 
268 methods. The OPLEC $_{m}$ calibration model with five underlying components provided the best predictive 269 results with a RMSEP test value as low as 0.005 , while the corresponding best $\mathrm{RMSEP}_{\text {test }}$ value of the 270 PLS calibration model with nine underlying components on the calibration spectra pre-processed by 271 EISC is 0.009. Furthermore, the performance of the $\mathrm{OPLEC}_{\mathrm{m}}$ is robust to the number of columns in $\mathbf{U}_{\mathrm{s}}$ 272 (Figure 3b). Considering the fact that $\mathrm{OPLEC}_{\mathrm{m}}$ does not place any extra requirement on the spectral 273 measurements as other multiplicative effect correction methods do, such a result is quite encouraging. 

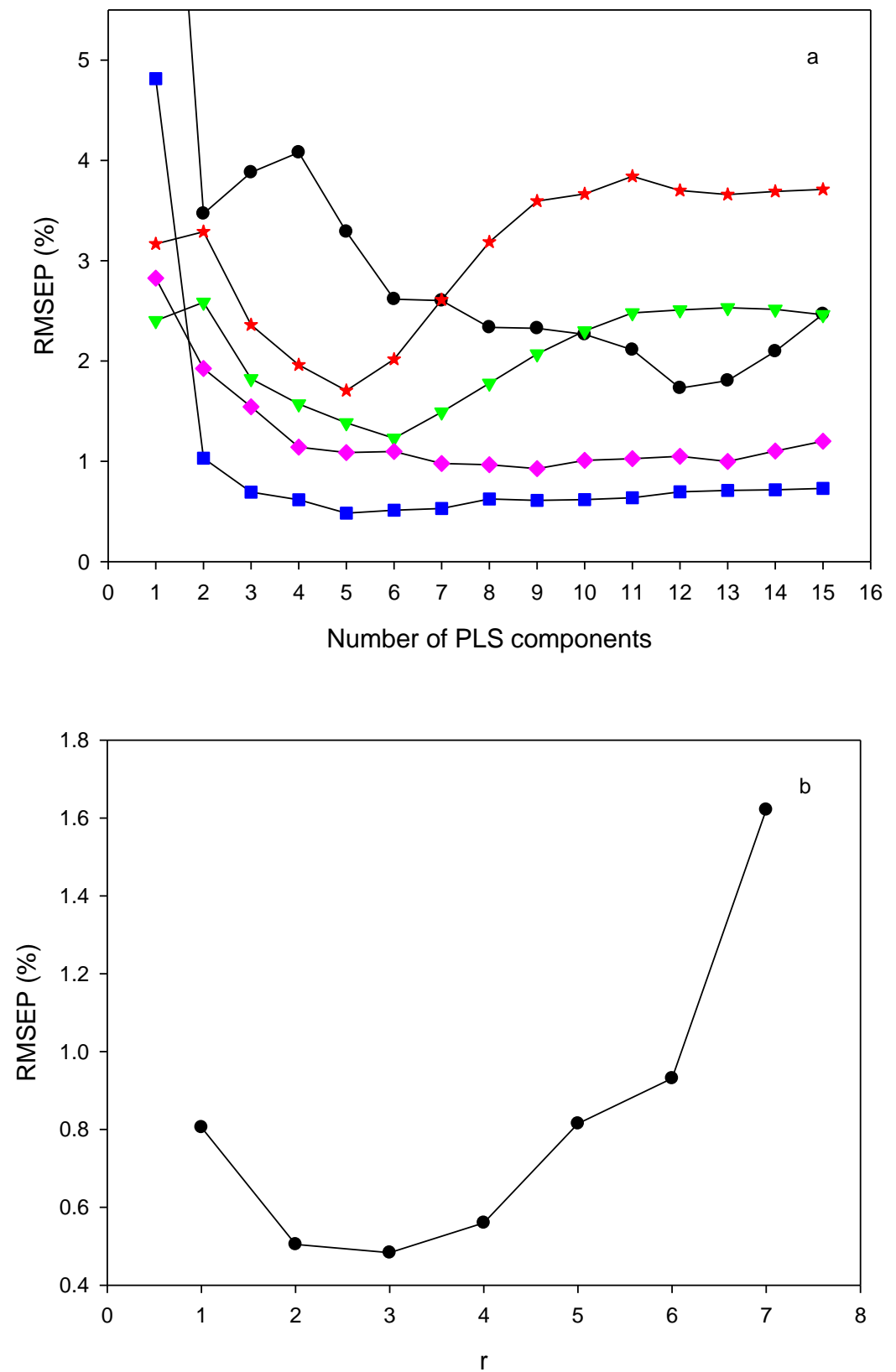

Figure 3: a) The predictive performance of OPLEC $\mathrm{m}_{\mathrm{m}}$ and the PLS models built on the calibration spectra of the four component suspension system preprocessed by different methods (black circle: the raw spectra; red star: MSC; green triangle down: SNV; pink diamond: EISC; blue square: OPLEC $\mathrm{m}_{\mathrm{m}}$ ); b) The predictive performance of the optimal OPLEC $_{\mathrm{m}}$ models when $\mathbf{U}_{s}$ with different number of columns $(r)$ were used in the calculation of the multiplicative parameter vector $\mathbf{p}$ for the calibration spectra. 
283 As in four component suspension data, there are significant additive baseline effects in the tecator data 284 (supporting information, Figure S-2). Since the changes in physical properties of samples generally 285 result in both additive baseline effects and multiplicative effects, the presence of significant additive 286 baseline effects strongly suggests the existence of multiplicative effects. OPLEC $m$ was therefore used to 287 estimate the multiplicative parameter vector $\mathbf{p}$ for the calibration samples from the corresponding 288 orthogonal projection pre-processed calibration spectra as described in section 3.3. During the 289 estimation of the multiplicative parameter vector $\mathbf{p}$ for the calibration samples using OPLEC $\mathrm{m}_{\mathrm{m}}$, the 290 optimal number of columns included in $\mathbf{U}_{\text {s }}$ (i.e. $r$ ) is determined by scrutinizing the plot of $\min _{\mathbf{p}} f(\mathbf{p})$ 291 versus $r$ (Figure 4). It can be seen that $\min _{\mathbf{p}} f(\mathbf{p})$ drops sharply as the $r$ increases from one to six, and 292 then decreases slowly along with the further increase of $r$ (Figure 4). One can therefore choose six as the 293 optimal number of columns of $\mathbf{U}_{s}$. 


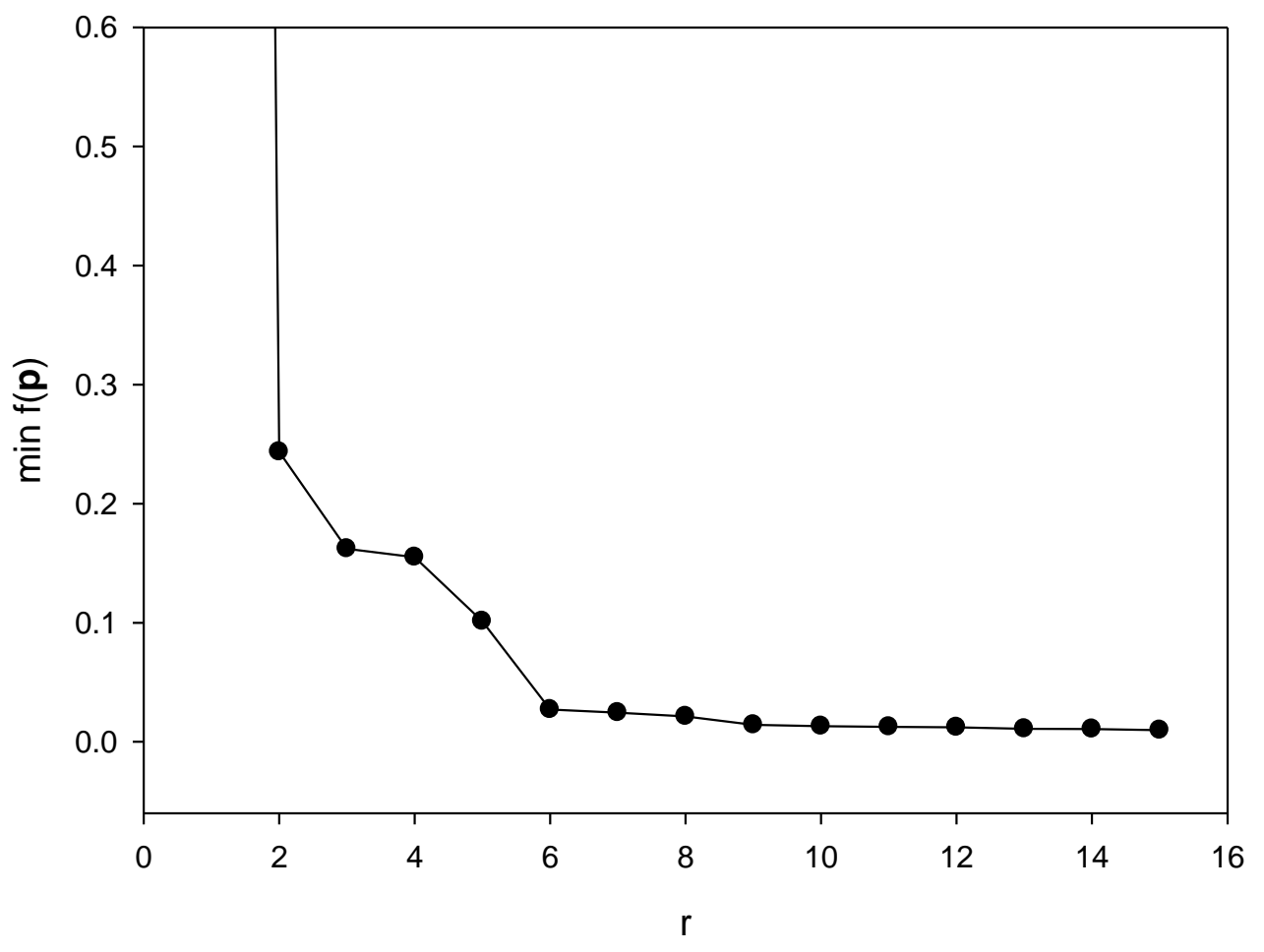

294

295 Figure 4: The plot of $\min _{\mathbf{p}} f(\mathbf{p})$ versus the number of columns in $\mathbf{U}_{s}$ (i.e. $r$ ). 
296 It is worth to point out again that the performance of OPLEC $\mathrm{m}_{\mathrm{m}}$ is quite robust to the choice of $r$ as long 297 as $r$ is big enough but not too large. As shown in Figure 5, The RMSEP value of OPLEC $\mathrm{m}_{\mathrm{m}}$ for the test 298 samples shows no significant difference when $r$ taking a value between 6 and 11. In practice, such a 299 feature of OPLEC $\mathrm{m}_{\mathrm{m}}$ can make it more user-friendly when being applied to complex systems. 


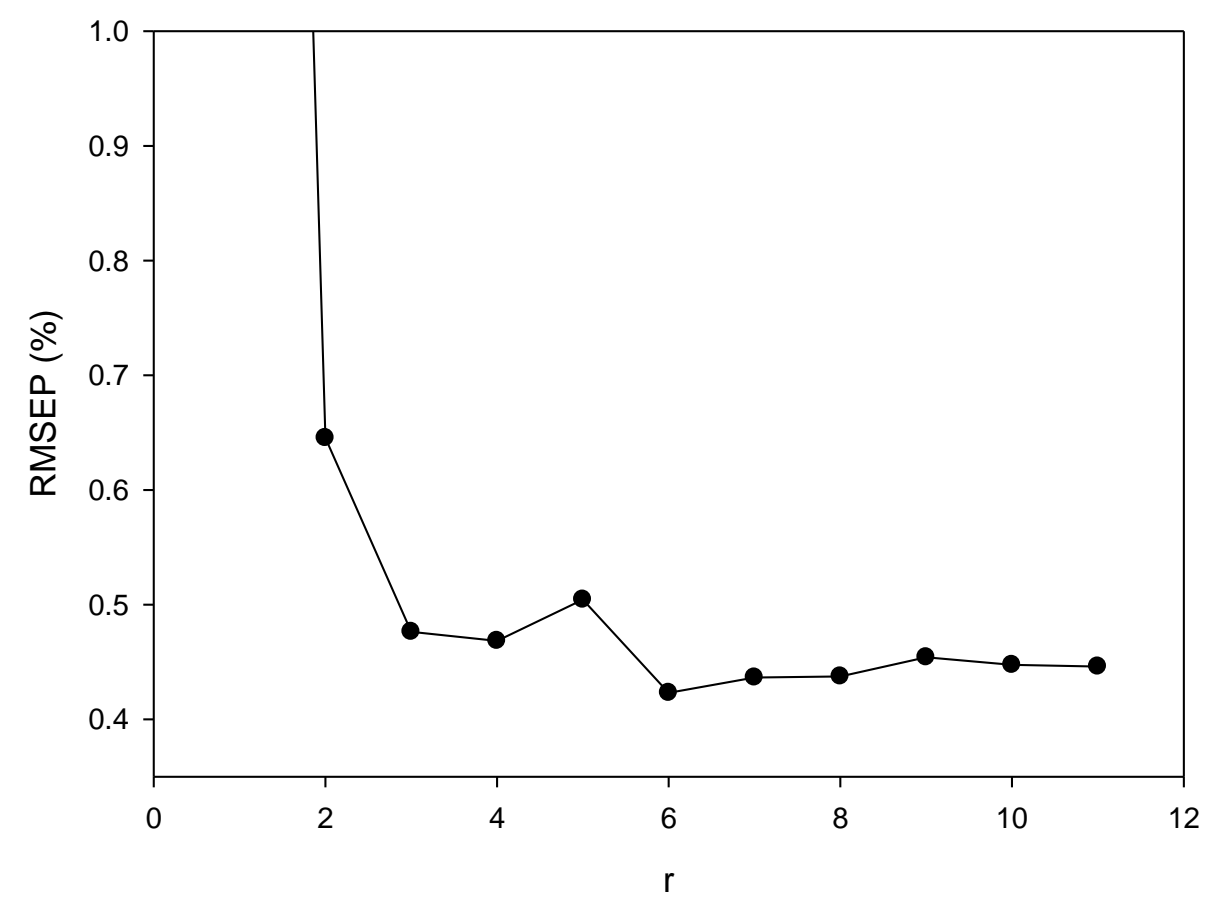

301 Figure 5: The RMSEP values for the test samples in the tecator data obtained by the optimal OPLEC $\mathrm{m}$ 302 calibration models when $\mathbf{U}_{s}$ with different number of columns (i.e. $r$ ) were used in the calculation of the 303 multiplicative parameter vector $\mathbf{p}$ for the calibration spectra. 
305 After the estimation of the multiplicative parameter vector $\mathbf{p}$ for the calibration samples, the dual 306 calibration strategy of OPLEC $\mathrm{m}_{\mathrm{m}}$ was adopted to mitigate the detrimental of multiplicative effects on the 307 prediction of the fat content. PLS calibration models with and without the application of MSC, SNV and 308 EISC were also established for comparison purposes. The optimal number of underlying components 309 used in the dual calibration models of OPLEC as well as those PLS calibration models was chosen to be 310 the one with minimal root-mean-square error of prediction (RMSEP) for the validation set. The results 311 of OPLEC $_{\mathrm{m}}$ along with those of the four optimal PLS calibration models with and without the 312 application of MSC, SNV and EISC were shown in Figure 6. 


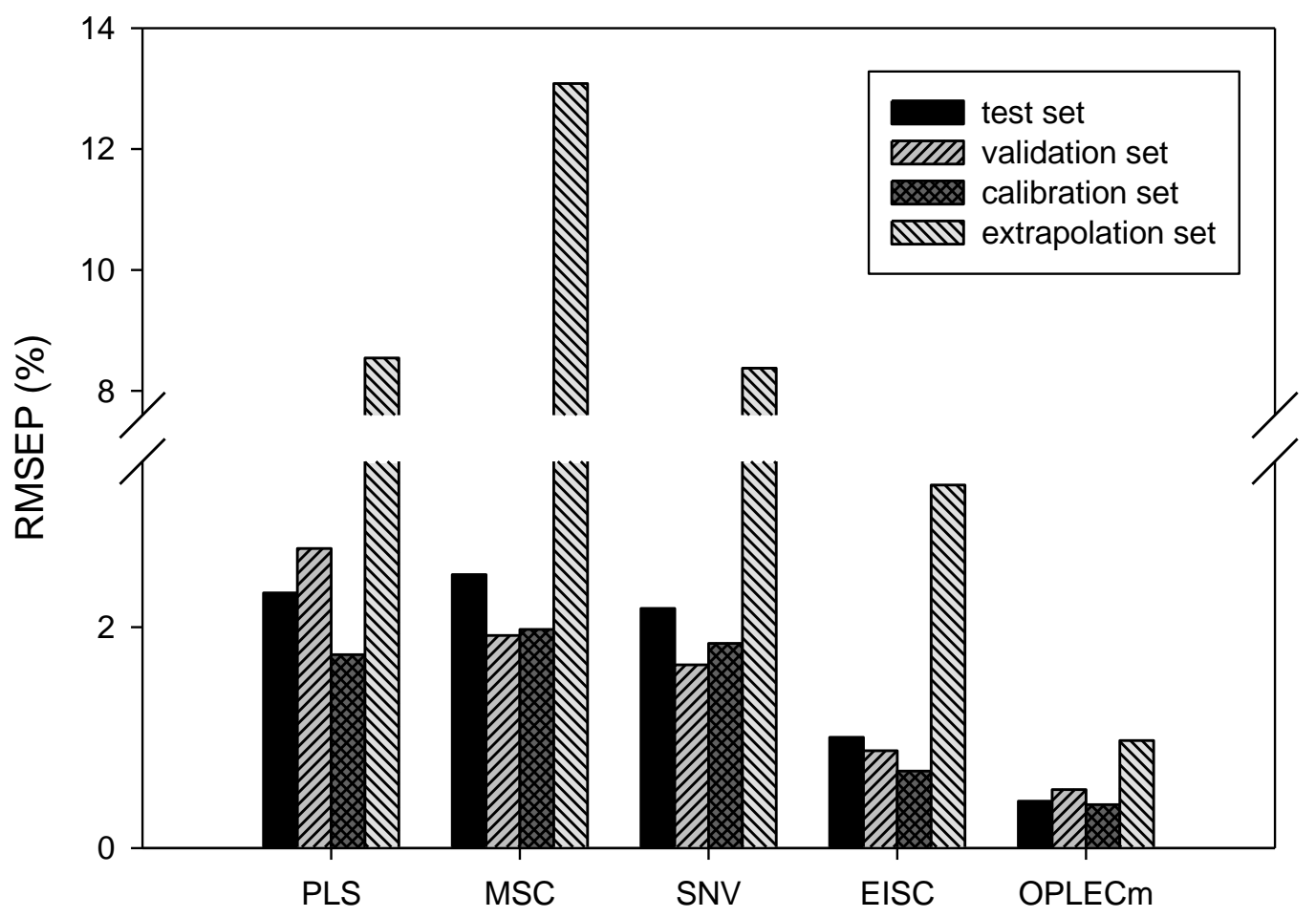

313

314 Figure 6: The RMSEP values for the tecator data obtained by different calibration methods. 
315 Figure 6 reveals that although the number of latent components (i.e. fourteen) used is sufficiently

316 large, the optimal PLS calibration model on the raw calibration spectra did not give satisfactory

317 predictions for all the four data sets. The RMSEP values for the calibration, validation, test and

318 extrapolation sets are $1.7 \%, 2.7 \%, 2.3 \%$ and $8.5 \%$, respectively. The application of the empirical

319 multiplicative light scattering correction method, SNV saw no significant changes in the RMSEP values

320 for the four data sets. While preprocessing the spectral data by MSC resulted in a dramatic increase in

321 the RMSEP value for the extrapolation set which clearly demonstrates its limitation in practical

322 applications. The EISC preprocessing method surprisingly succeeded in improving the quality of the

323 predictions of PLS calibration model for the tecator data. Its RMSEP values for the calibration,

324 validation, test and extrapolation sets are $0.7 \%, 0.9 \%, 1.0 \%$ and $3.3 \%$, respectively. The reasons of its

325 success in this particular data set are unclear. As expected, OPLEC $_{m}$ outperformed all the other methods

326 with RMSEP values for the calibration, validation, test and extrapolation sets equaling to $0.4 \%, 0.5 \%$,

$3270.4 \%$ and $1.0 \%$, respectively, This remarkable improvement further confirmed the effectiveness of

$328 \mathrm{OPLEC}_{\mathrm{m}}$ in mitigating the detrimental influence of multiplicative effects on the spectroscopic 329 quantitative analysis of heterogeneous mixture samples.

\section{Conclusion}

333 The separation of the spectral contributions due to variations in chemical compositions from

334 multiplicative effects caused by physical variations is crucial to the accurate quantitative analysis of

335 complex heterogeneous mixture samples using spectroscopic instruments. In this work, a modified 
336 version of Optical Path-Length Correction and Estimation $\left(\right.$ OPLEC $\left._{\mathrm{m}}\right)$ method has been developed to

337 correct the multiplicative effects in spectral measurements. OPLEC $\mathrm{m}_{\mathrm{m}}$ differs from the original OPLEC

338 method in the way of estimating the multiplicative parameters for the calibration samples. In OPLEC $\mathrm{m}_{\mathrm{m}}$,

339 the multiplicative parameters for the calibration samples were obtained by solving a constrained

340 quadratic programming problem, which is much more efficient than the counterpart in the original

341 OPLEC. Furthermore, a simple but effective method has been proposed for the determination of the

342 model parameter involved (i.e. the number of spectroscopically active chemical components in the

343 system under study). Due to the unique multiplicative parameter estimation strategy, the performance of

344 OPLEC $_{\mathrm{m}}$ is much more robust to the choice of the model parameter involved, which makes OPLEC $\mathrm{m}$

345 more user-friendly when being applied to complex systems. The performance of $\mathrm{OPLEC}_{\mathrm{m}}$ has been

346 tested on four-component suspension spectral data set and one publicly available benchmark spectral

347 data set. Experimental results reveal that $\mathrm{OPLEC}_{\mathrm{m}}$ can achieve satisfactory quantitative results from the

348 spectroscopic measurements of heterogeneous mixtures. Compared with other existing methods

349 designed for the same purpose, OPLEC $_{\mathrm{m}}$ has features of implementation simplicity, wider applicability

350 as well as better performance in terms of quantitative accuracy, and therefore has great potential in

351 quantitative spectroscopic analysis of complex heterogeneous systems. 
358 The authors acknowledge the financial support of the National Natural Science Foundation of China 359 (grant no. 21075034), “973” National Key Basic Research Program of China (grant no. 2007CB310500) 360 and the Fundamental Research Funds for the Central Universities of China and also Marie Curie FP6 361 (INTROSPECT)..

\section{Supporting Information Available}

365 MATLAB code for the modified OPLEC, the plots of $\mathbf{p}$ vs $\mathbf{U}_{s} \mathbf{U}_{s}^{T} \mathbf{p}$ and $\operatorname{diag}\left(\mathbf{c}_{1}\right) \mathbf{p} \quad \operatorname{vs} \mathbf{U}_{s} \mathbf{U}_{s}^{T} \operatorname{diag}\left(\mathbf{c}_{1}\right) \mathbf{p}$

366 for the four component suspension data, the 129 raw calibration spectra of the tecator data. This material 367 is available free of charge via the Internet at http://pubs.acs.org.

368 


\section{References :}

371 (1) H.W. Siesler, Y. Ozaki, S. Kawata, H.M. Heise, Near-infrared spectroscopy: principal, 372 instruments, applications, WILEY-VCH, Weinheim, 2002

373 (2) P. Fayolle, D. Picque, G. Corrieu, Monitoring of fermentation processes producing lactic acid 374

(3) Y. Roggo, C. Roeseler, M. Ulmschneider, Near infrared spectroscopy for qualitative comparison of pharmaceutical batches, J. Pharm. Biomed. Anal. 2004, 36, 777-786

(6) Z.P. Chen, J. Morris, A. Borissova, S. Khan, T. Mahmud, R. Penchev, K.J. Roberts, On-line monitoring of batch cooling crystallization of organic compounds using ATR-FTIR spectroscopy coupled with an advanced calibration method, Chemom. Intell. Lab. Syst. 2009, 96, 49-58

(7) P. Geladi, D. MacDougall, H. Martens, Linearization and Scatter-Correction for Near-Infrared Reflectance Spectra of Meat, Appl. Spectrosc. 1985, 39 (3), 491-500 
388 (8) I.A. Cowe, J.W. McNicol, The Use of Principal Components in the Analysis of Near-Infrared Spectra, Appl. Spectrosc. 1985, 39 (2), 257-266

(9) H. Martens, M. Martens, Multivariate Analysis of Quality: An Introduction, John Wiley and Sons: Chichester, 2001

(10) R.J. Barnes, M.S. Dhanoa, S.J. Lister, Standard Normal Variate Transformation and De-trending of Near-Infrared Diffuse Reflectance Spectra, Appl. Spectrosc. 1989, 43 (5), 772-777

(11) I.S. Helland, T. Næs, T. Isaksson, Related versions of the multiplicative scatter correction method for preprocessing spectroscopic data, Chemom. Intell. Lab. Syst. 1995, 29 (2), 233-241

(12) D. Pedersen, H. Martens, J. Nielsen, S. Engelsen, Near-infrared absorption and scattering separated by extended inverted signal correction (EISC): Analysis of near-infrared transmittance spectra of single wheat seeds, Appl. Spectrosc. 2002, 56 (9), 1206-1214

(13) H. Martens, J.P. Nielsen, S.B. Engelsen, Light Scattering and Light Absorbance Separated by Extended Multiplicative Signal Correction. Application to Near-Infrared Transmission Analysis of Powder Mixtures, Anal. Chem. 2003, 75 (3), 394-404

(14) S.N. Thennadil, H. Martens, A. Kohler, Physics-based multiplicative scatter correction approaches for improving the performance of calibration models, Appl. Spectrosc. 2006, 60, 315-321

(15) R. Steponavicius, S.N. Thennadil, Extraction of chemical information of suspensions using radiative transfer theory to remove multiple scattering effects: application to a model two-component system, Anal. Chem. 2009, 81, 7713-7723 
407 (16) R. Steponavicius, S.N. Thennadil, Extraction of chemical information of suspensions using 408 Radiative transfer theory to remove multiple scattering effects: application to a model 409 multicomponent system, Anal. Chem. 2011, 83, 1931-1937

410 (17) Z. Shi, C. Andersen, Pharmaceutical applications of separation of absorption and scattering in 411 near-infrared spectroscopy (NIRS), J. Pharm. Sci. 2010, 99, 4766-4783

412 (18) W. Kessler, D. Oelkrug, R. Kessler, Using scattering and absorption spectra as MCR-hard model 413 constraints for diffuse reflectance measurements of tablets, Anal. Chim. Acta, 2009, 642, 127-134

414 (19) Z.P.Chen, J. Morris, E. Martin, Extracting Chemical Information from Spectral Data with 415 Multiplicative Light Scattering Effects by Optical Path-Length Estimation and Correction, Anal. $416 \quad$ Chem. 2006, 78(9), 7674-7681

417 (20) Z.P.Chen, L.J. Zhong, A. Nordon, D. Littlejohn, M. Holden, M. Fazenda, L. Harvey, B. McNeil, 418 J. Faulkner, J. Morris, Calibration of Multiplexed Fiber-Optic Spectroscopy, Anal. Chem. 2011, $419 \quad 83(7), 2655-2659$

420 (21) Z.P. Chen, J. Morris, Improving the linearity of spectroscopic data subjected to fluctuations in 421 external variables by the extended loading space standardization, The Analyst, 2008, 133, 914-922

422 (22) C. Borggaard, H.H. Thodberg, Optimal minimal neural interpretation of spectra, Anal. Chem. 423 1992, $64,545-551$ 


Heterogeneous Mixtures


3 Title of the primary article:

4 Quantitative Spectroscopic Analysis of Heterogeneous Mixtures: the Correction of Multiplicative 5 Effects Caused by Variations in Physical Properties of Samples

\section{Authors' names:}

7 Jing-Wen Jin $^{a}$, Zeng-Ping Chen* ${ }^{a}$, Li-Mei Li ${ }^{a}$, Raimundas Steponavicius ${ }^{b}$, Suresh N. Thennadil ${ }^{c}, J_{i n g}$ 8 Yang $^{a}$ and Ru-Qin $Y u^{*^{a}}$

\section{Affiliations:}

10 a. State Key Laboratory of Chemo/Biosensing and Chemometrics, College of Chemistry and Chemical 11 Engineering, Hunan University, Changsha, Hunan, 410082, PR China

12 b. School of Chemical Engineering and Advanced Materials, Newcastle University, Merz Court, 13 Newcastle upon Tyne, NE1 7RU, United Kingdom

c. Chemical and Process Engineering, University of Strathclyde, 75 Montrose Street, Glasgow, G1 1XJ, 5 United Kingdom

16 Table of content:

17 1) The MATLAB code for the modified OPLEC method

18 2) Figure S-1: The plots of $\mathbf{p}$ vs $\mathbf{U}_{s} \mathbf{U}_{s}^{\mathrm{T}} \mathbf{p}$ (a) and $\operatorname{diag}\left(\mathbf{c}_{1}\right) \mathbf{p}$ vs $\mathbf{U}_{s} \mathbf{U}_{s}^{\mathrm{T}} \operatorname{diag}\left(\mathbf{c}_{1}\right) \mathbf{p}$ (b) for the four 19 component suspension data.

20 3) Figure S-2: The 129 raw calibration spectra of the tecator data. 
$23 \% \quad[\mathbf{p}, \mathrm{fval}]=\mathrm{OPLEC}_{\mathrm{m}}(\mathbf{X}, \mathbf{c}$, CompNumb $)$

$24 \%$ This is an $\mathrm{m}$-file for the estimation of the multiplicative effect vector $\mathbf{p}$ for calibration samples;

$25 \% \mathbf{X}$ contains $\mathbf{x}_{i}$ in its rows; $\mathbf{x}_{i}(i=1,2, \cdots, I)$ are the spectra of $I$ calibration samples.

$26 \% \mathbf{c}$ is the concentration vector of the target chemical component in the calibration samples;

$27 \%$ CompNumb is the number of spectroscopically active chemical components in mixture samples;

$28 \% \mathbf{p}$ is a vector containing the multiplicative scattering parameters for the calibration samples;

$29 \%$ fval is the value of objective function at $\mathbf{p}$;

30

31 function $[\mathbf{p}$, fval $]=\operatorname{OPLECm}(\mathbf{X}, \mathbf{c}, \mathrm{CompNumb})$;

$32 \quad[\mathbf{U}, \mathbf{S}, \mathbf{V}]=\operatorname{svd}(\mathbf{X})$

$33 \quad \mathbf{U s}=\mathbf{U}(:, 1:$ CompNumb $)$;

$34 n=$ length $(\mathbf{c})$;

$35 \quad w=\max (\mathbf{c})$;

$36 \quad \mathbf{H} 1=$ eye(n, n)- Us* Us';

$37 \quad \mathbf{H} 2=\operatorname{diag}(\mathbf{c} . / w) * \mathbf{H} 1 * \operatorname{diag}(\mathbf{c} . / w) ;$ 
$38 \quad \mathbf{H}=\mathbf{H} 1+\mathbf{H} 2 ; \quad \%$ matrix $\mathbf{H}$ in $\min \left(0.5^{*} \mathbf{p}^{* *} \mathbf{H}^{*} \mathbf{p}+\mathbf{f}^{*} \mathbf{p}\right)$

$39 \mathbf{f}=$ zeros $(\mathrm{n}, 1) ; \quad \%$ vector $\mathbf{f}$ in $\min \left(0.5^{*} \mathbf{p}^{\prime *} \mathbf{H}^{*} \mathbf{p}+\mathbf{f}^{*} \mathbf{p}\right)$;

$40 \quad \mathbf{A}=-$ eye $(n, n) ; \quad \%$ matrix $\mathbf{A}$ in $\mathbf{A}^{*} \mathbf{p}<=\mathbf{b}$;

$41 \quad \mathbf{b}=-$ ones $(\mathrm{n}, 1) ; \quad \%$ vector $\mathbf{b}$ in $\mathbf{A}^{*} \mathbf{p}<=\mathbf{b}$;

$42 \quad$ StartingVect $=$ ones $(n, 1)$;

43 options=optimset('quadprog');

44 options=optimset(options,'LargeScale','off','Display','off');

$45 \quad[\mathbf{p}$, fval] $]=$ quadprog $(\mathbf{H}, \mathbf{f}, \mathbf{A}, \mathbf{b},[],[],[],[]$, StartingVect,options $)$;

$46 \%$ After obtaining the model parameter vector $\mathbf{p}$ for calibration samples, two calibration models are built

47 using the standard PLS toolbox. One is between the concentration vector (c) of the target chemical

48 component and the spectral data $\mathbf{X}$; the other is between $\operatorname{diag}(\mathbf{c}) \mathbf{p}$ and $\mathbf{X}$. The multiplicative effect on

49 the test sample can then be corrected through dividing the prediction of the second calibration model by

50 the prediction of the first calibration model. 
51 1) Figure S-1: The plots of $\mathbf{p}$ vs $\mathbf{U}_{s} \mathbf{U}_{s}^{T} \mathbf{p}$ (a) and $\operatorname{diag}\left(\mathbf{c}_{1}\right) \mathbf{p}$ vs $\mathbf{U}_{s} \mathbf{U}_{s}^{T} \operatorname{diag}\left(\mathbf{c}_{1}\right) \mathbf{p}$ (b) for the four 52 component suspension data. The number of columns in $U_{s}$ is three.

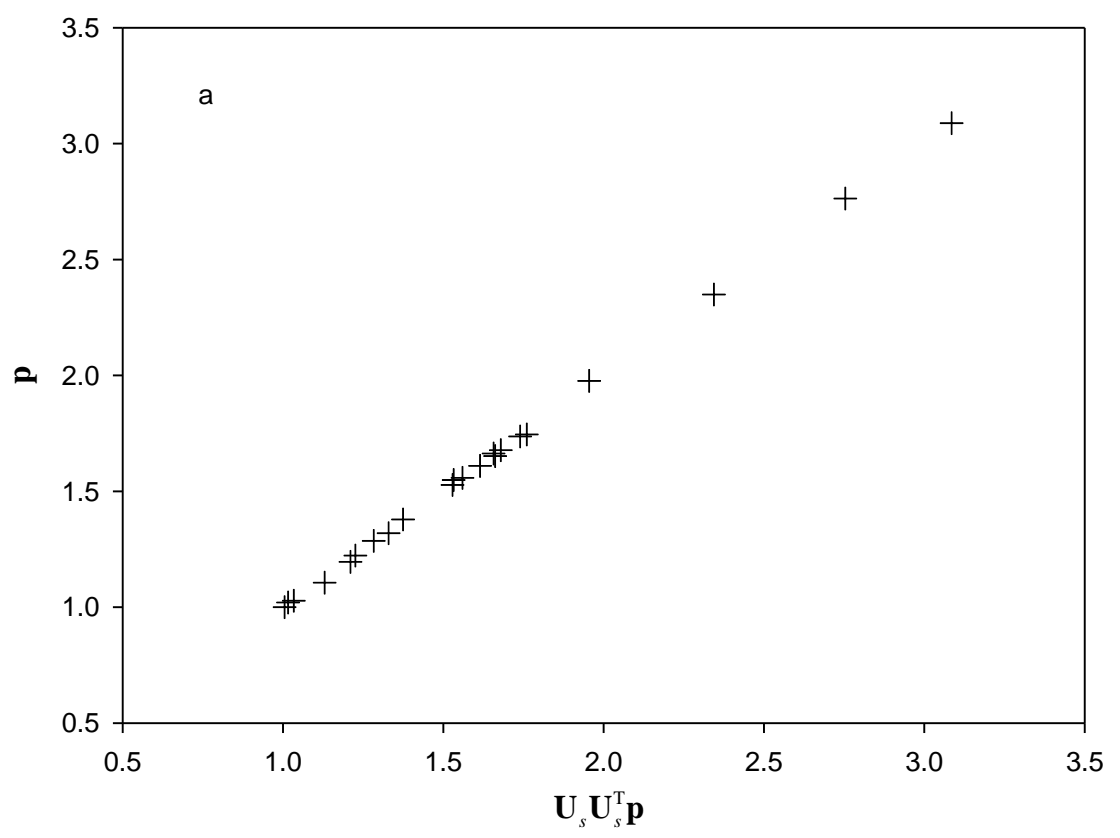

53

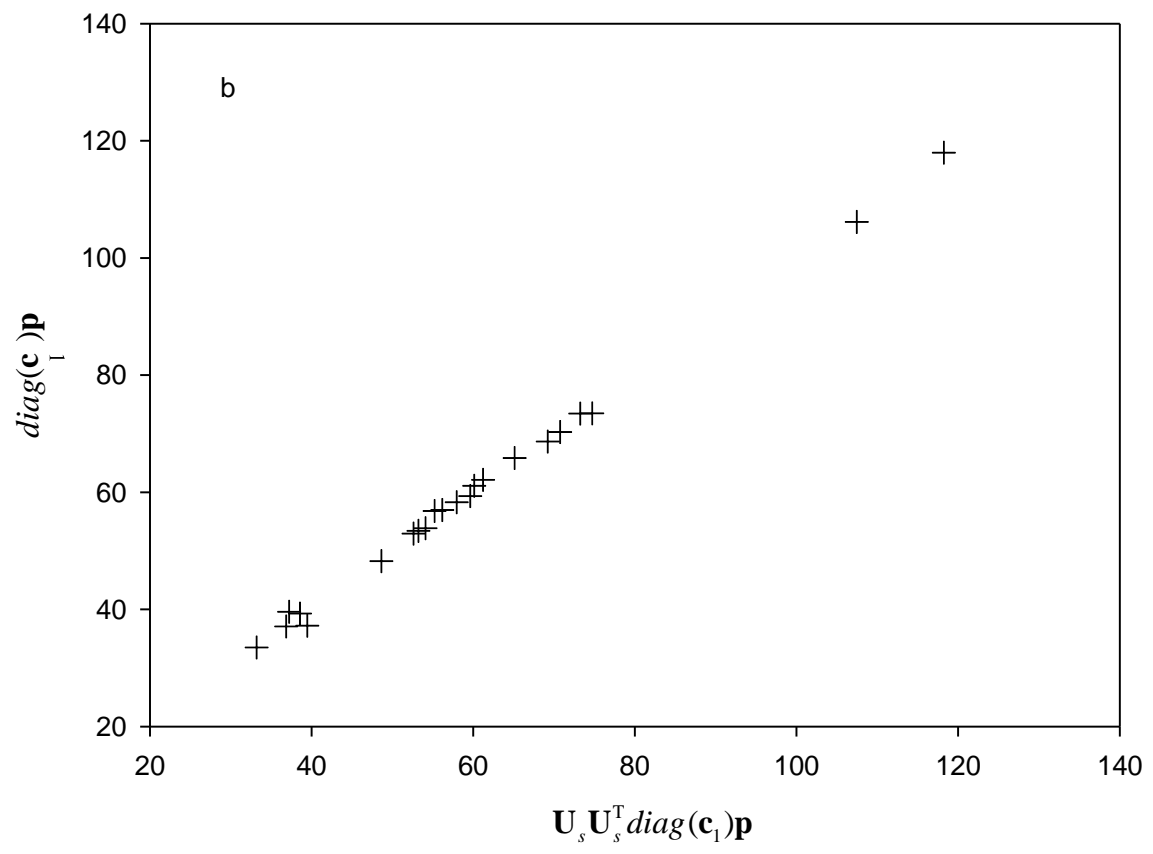


55 2) Figure S-2: The 129 raw calibration spectra of the tecator data.

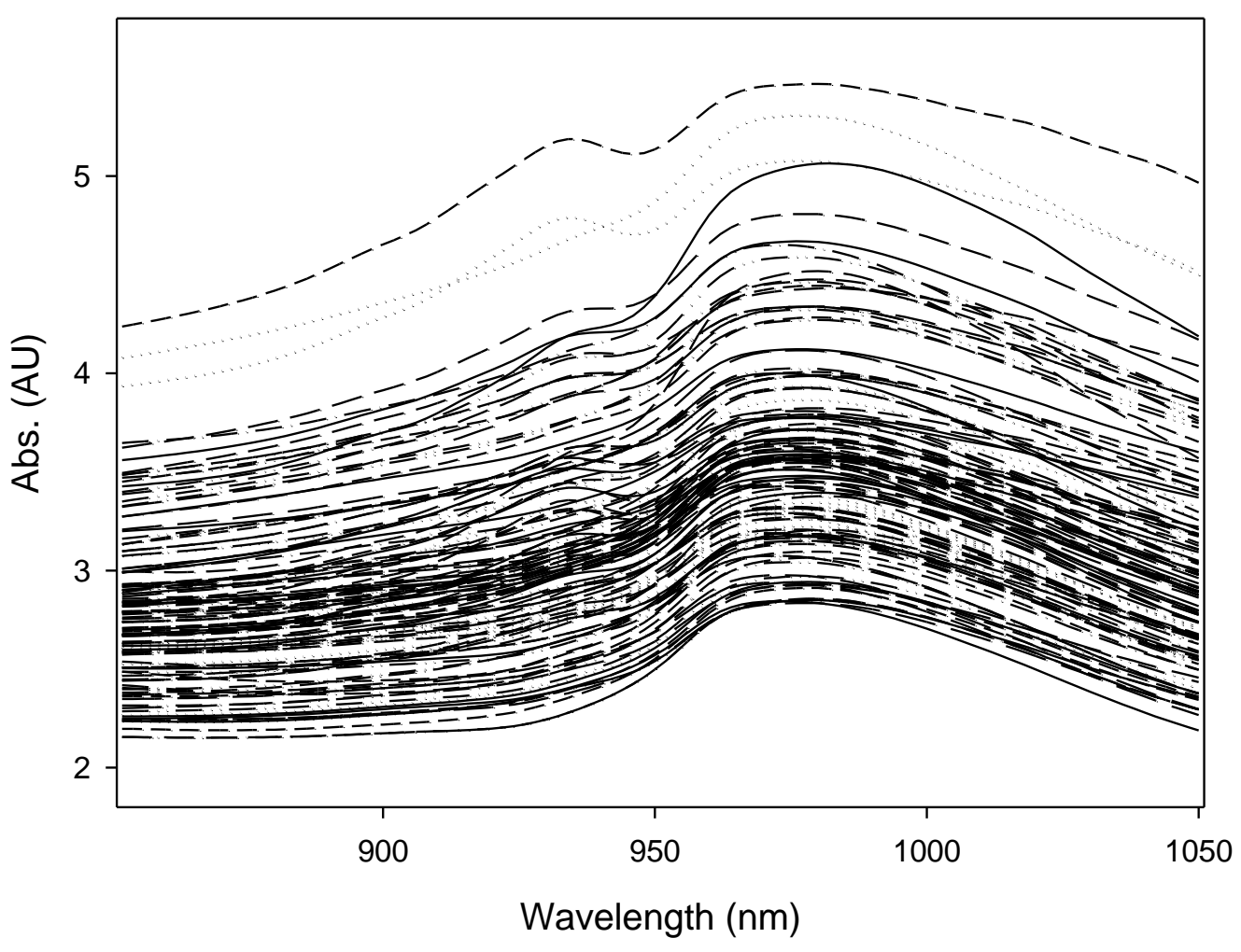

